\title{
A Nonsimple Conjoint Measurement Model
}

\author{
LILY C. HUANG*
}

The University of Michigan, Ann Arbor, Michigan

\begin{abstract}
The nonsimple conjoint measurement model examined in this paper maps each $\left(a_{1}, a_{2}, a_{3}\right)$ in $A_{1} \times A_{2} \times A_{3}$ into $\omega_{3}\left(a_{1}\right) \phi_{2}\left(a_{2}\right)+\omega_{2}\left(a_{1}\right) \phi_{3}\left(a_{3}\right)$, where each of $\omega_{1}, \omega_{2}$, $\phi_{2}$, and $\phi_{3}$ is a real-valued function, so as to preserve a binary relation $\geqslant$ on $A_{1} \times A_{2} \times A_{3}$ by $\geqslant$ in the numerical system.

The principle structure of the present model is similar to the structure of the simple models. But in the nonsimple model, we define two different identity elements of $A_{1}$ for its different multiplicative effects on the other two components $A_{2}$ and $A_{3}$, whereas in the simple model, one identity element for each component is defined.
\end{abstract}

\section{INTRODUCTION}

The development of simple conjoint measurement models (Krantz, Luce, Suppes, and Tversky, 1971) makes real-valued scales for psychological measurement possible under certain conditions and offers a measurement-free technique in testing hypotheses about such composition rules. Some models hypothesized in psychology are not simple composition rules so the development of axiom systems for nonsimple models will offer measurement-free techniques for testing such theories.

An axiom system for a familiar but nonsimple model will be presented. Hopefully, the principle of these axiom systems can be extended to construct more psychological models.

The nonsimple conjoint measurement model examined in this paper maps each $\left(a_{1}, a_{2}, a_{3}\right)$ in $A_{1} \times A_{2} \times A_{3}$ into $\omega_{1}\left(a_{1}\right) \phi_{2}\left(a_{2}\right)+\omega_{2}\left(a_{1}\right) \phi_{3}\left(a_{3}\right)$, where each of $\omega_{1}, \omega_{2}$, $\phi_{2}$, and $\phi_{3}$ is a real-valued function, so as to preserve a binary relation $\geqslant$ on $A_{1} \times A_{2} \times A_{3}$ by $\geqslant$ in the numerical system.

The principle structure of the present model is similar to the structure of the simple models. But in the nonsimple model, we define two different identity elements of $A_{1}$ for its different multiplicative effects on the other two components $A_{2}$ and $A_{3}$, whereas in the simple model, one identity element for each component is defined. The principle

* This research was supported by National Science Foundation Grant GB-15653 to the University of Michigan. The author expresses her gratitude to Professors Clyde H. Coombs and David H. Krantz for their enormous contributions to the formation of this paper. 
of using multiple identity elements for one component can be generalized to construct the axiom systems for the more complicated models, e.g., $\omega_{1}\left(a_{1}\right) \phi_{2}\left(a_{2}\right)+$ $\omega_{2}\left(a_{2}\right) \phi_{3}\left(a_{3}\right)+\omega_{3}\left(a_{1}\right) \phi_{4}\left(a_{4}\right)$.

\section{Definitions}

Definition $1 . \geqslant$ is a binary relation on $A_{1} \times A_{2} \times A_{3}$. Two elements $\left(a_{1}, a_{2}, a_{3}\right)$ and $\left(b_{1}, b_{2}, b_{3}\right)$ of $A_{1} \times A_{2} \times A_{3}$ are equivalent, $\left(a_{1}, a_{2}, a_{3}\right) \sim\left(b_{1}, b_{2}, b_{3}\right)$ if and only if they satisfy the following two conditions:

$$
\left(a_{1}, a_{2}, a_{3}\right) \geqslant\left(b_{1}, b_{2}, b_{3}\right)
$$

and

$$
\left(b_{1}, b_{2}, b_{3}\right) \geqslant\left(a_{1}, a_{2}, a_{3}\right) .
$$

We first define the cancellation condition known as the Thomson condition.

Definition 2. A relation $\sim$ on $\left\{a_{1}\right\} \times A_{2} \times A_{3}$, where $a_{1} \in A_{1}$, satisfies the Thomsen condition provided that for every $a_{2}, b_{2}, k_{2} \in A_{2}$ and $a_{3}, b_{3}, k_{3} \in A_{3}$, if

$$
\left(a_{1}, a_{2}, k_{3}\right) \sim\left(a_{1}, k_{2}, b_{3}\right)
$$

and

$$
\left(a_{1}, k_{2}, a_{3}\right) \sim\left(a_{1}, b_{2}, k_{3}\right),
$$

then

$$
\left(a_{1}, a_{2}, a_{3}\right) \sim\left(a_{1}, b_{2}, b_{3}\right) .
$$

The Thomsen condition is a necessary condition for the simple additive model. It is clear that the nonsimple model examined is an addtive model on $\left\{a_{1}\right\} \times A_{2} \times A_{3}$.

Definition 3. (1) $A_{2}{ }^{0}$ and $A_{3}{ }^{0}$ are the subsets of $A_{2}$ and $A_{3}$, respectively, such that if $a_{2}{ }^{0}, b_{2}{ }^{0} \in A_{2}{ }^{0}$ and $a_{3}{ }^{0}, b_{3}{ }^{0} \in A_{3}{ }^{0}$, then

$$
\begin{aligned}
& \left(a_{1}, a_{2}{ }^{0}, a_{3}\right) \sim\left(a_{1}, b_{2}{ }^{0}, a_{3}\right) \quad \text { for all } a_{1} \in A_{1} \text { and } a_{3} \in A_{3} \text {; } \\
& \left(a_{1}, a_{2}, a_{3}{ }^{0}\right) \sim\left(a_{1}, a_{2}, b_{3}{ }^{0}\right) \text { for all } a_{1} \in A_{1} \text { and } a_{2} \in A_{2} \text {; } \\
& \left(a_{1}, a_{2}{ }^{0}, a_{3}{ }^{0}\right) \sim\left(b_{1}, a_{2}{ }^{0}, a_{3}{ }^{0}\right) \quad \text { for all } a_{1}, b_{1} \in A_{1} \text {. }
\end{aligned}
$$

(2) $A_{1}{ }^{0}$ and $A_{1}^{00}$ are the subsets of $A_{1}$, such that if $a_{1}{ }^{0} \in A_{1}{ }^{0}$ and $a_{1}^{00} \in A_{1}^{00}$, then

$$
\begin{aligned}
& \left(a_{1}{ }^{0}, a_{2}, a_{3}{ }^{0}\right) \sim\left(a_{1}{ }^{0}, b_{2}, a_{3}{ }^{0}\right) \quad \text { for all } a_{2}, b_{2} \in A_{2} \quad \text { and } \quad a_{3}{ }^{0} \in A_{3}{ }^{0} \text {; } \\
& \left(a_{1}^{00}, a_{2}^{0}, a_{3}\right) \sim\left(a_{1}^{00}, a_{2}^{0}, b_{3}\right) \quad \text { for all } a_{3}, b_{3} \in A_{3} \text { and } a_{2}{ }^{0} \in A_{2}{ }^{0} \text {. }
\end{aligned}
$$


$A_{2}{ }^{0}$ and $A_{3}{ }^{0}$ are the sets of zero elements of $A_{2}$ and $A_{3}$, respectively. $A_{1}{ }^{0}$ and $A^{00}$ are two sets of zero elements of $A_{1}$ and have degenerate effects on $A_{2}$ and $A_{3}$, respectively.

Definition 4. (1) $\left\{a_{1}\right\} \times A_{2}$ is independent of $\left\{a_{1}\right\} \times A_{3}$ if and only if for $a_{2}, b_{2} \in A_{2},\left(a_{1}, a_{2}, x_{3}\right) \geqslant\left(a_{1}, b_{2}, x_{3}\right)$ for some $x_{3} \in A_{3}$ implies that $\left(a_{1}, a_{2}, y_{3}\right) \geqslant$ $\left(a_{1}, b_{2}, y_{3}\right)$ for every $y_{3} \in A_{3}$.

$\left\{a_{1}\right\} \times A_{2}$ and $\left\{a_{1}\right\} \times A_{3}$ are mutually independent if and only if $\left\{a_{1}\right\} \times A_{2}$ is independent of $\left\{a_{1}\right\} \times A_{3}$ and $\left\{a_{1}\right\} \times A_{3}$ is independent of $\left\{a_{1}\right\} \times A_{2}$.

Definition 5. $A_{1}$ is sign dependent on $A_{2} \times A_{3}{ }^{0}$ if and only if $A_{2}$ can be partitioned into three sets, $A_{2}{ }^{+}, A_{2}{ }^{0}$, and $A_{2}{ }^{-}$such that for any $a_{1}, b_{1} \in A_{1}$ and $a_{3}{ }^{0} \in A_{3}{ }^{0}$ the following conditions hold.

\begin{tabular}{|c|c|c|}
\hline$\left(a_{1}, x_{2}, a_{3}^{0}\right) \geqslant\left(b_{1}, x_{2}, a_{3}^{0}\right)$ & for some & $x_{2} \in A_{2}^{+}$ \\
\hline$\left(a_{1}, y_{2}, a_{3}{ }^{0}\right) \geqslant\left(b_{1}, y_{2}, a_{3}{ }^{0}\right)$ & for every & $y_{2} \in A_{2}^{+}$ \\
\hline$\left(b_{1}, y_{2}, a_{3}^{0}\right) \geqslant\left(a_{1}, y_{2}, a_{3}^{0}\right)$ & for every & $y_{2} \in A_{2}^{-}$ \\
\hline$\left(a_{1}, x_{2}, a_{3}^{0}\right) \geqslant\left(b_{1}, x_{2}, a_{3}^{0}\right)$ & for some & $x_{2} \in A_{2}^{-}$ \\
\hline$\left(a_{1}, y_{2}, a_{3}{ }^{0}\right) \geqslant\left(b_{1}, x_{2}, a_{3}{ }^{0}\right)$ & for every & $y_{2} \in A_{2}^{-}$, \\
\hline$\left(b_{1}, y_{2}, a_{3}{ }^{0}\right) \geqslant\left(a_{1}, y_{2}, a_{3}{ }^{0}\right)$ & for every & $y_{2} \in A$ \\
\hline
\end{tabular}

$A_{1}$ and $A_{2} \times A_{3}{ }^{0}$ are mutually sign dependent if and only if $A_{1}$ is sign dependent on $A_{2} \times A_{3}{ }^{0}$ and $A_{2} \times A_{3}{ }^{n}$ is sign dependent on $A_{1}$.

Definition 6. For every pair of elements $a_{2}, b_{2} \in A_{2}, a_{2} \approx b_{2}$ if and only if $\left(a_{1}, a_{2}, a_{3}\right) \sim\left(a_{1}, b_{2}, a_{3}\right)$ for every $a_{1} \in A_{1}$ and $a_{3} \in A_{3} . A_{2}$ is essential if and only if there exist $a_{2}, b_{2} \in A_{2}$ such that $a_{2} \approx b_{2}$. Similar definition holds for $A_{3}$.

Definition 7. $\geqslant$ is a weak ordering of $\left\{a_{1}\right\} \times A_{2} \times A_{3}$. For any set $N$ of consecutive integers, $N=\{i, i+1, i+2, \ldots, j, j+1, j+2, \ldots\}$, a set $\left\{a_{2}{ }^{j} \mid a_{2}{ }^{j} \in A_{2}, j \in N\right\}$ is a standard sequence on $A_{2}$ iff there exist $x_{3} \not y_{3}$ such that $\left(a_{1}, a_{2}{ }^{j}, x_{3}\right) \sim\left(a_{1}, a^{j+1}, y_{3}\right)$ for all $j, j+1 \in N$. A standard sequence on $A_{2}$ is strictly bounded if and only if for any $a_{3} \in A_{3}$, there exist $\bar{a}_{2}$ and $\underline{\mathbf{a}}_{2}$ such that $\bar{a}_{2} \not \mathbf{a}_{2}$ and $\left(a_{1}, \bar{a}_{2}, a_{3}\right) \geqslant\left(a_{1}, a_{2}{ }^{j}, a_{3}\right) \geqslant$ $\left(a_{1}, a_{2}, a_{3}\right)$ for all $j \in N$. A bounded standard sequence is finite if and only if the integer set $N$ defined above is finite.

Thomsen condition, independence, sign dependence, and standard sequence were defined by Krantz et al. (Krantz, Luce, Suppes, and Tvresky, 1971). 
Definition 8. A relation $\sim$ on $A_{1} \times A_{2} \times A_{3}$ satisfies unrestricted solvability provided that for all $a_{2}{ }^{0} \in A_{2}{ }^{0}, a_{3}{ }^{0} \in A_{3}{ }^{0}, a_{1}, b_{1} \in A_{1}-A_{1}{ }^{0}-A_{1}^{00}, a_{2}, b_{2} \in A_{2}, a_{3}$, $b_{3} \in A_{3}$, there exist $b_{2}{ }^{\prime} \in A_{2}, b_{3}{ }^{\prime} \in A_{3}$ and $a_{1}{ }^{\prime}, a_{1}^{\prime \prime} \in A_{1}$ such that

$$
\begin{gathered}
\left(a_{1}, a_{2}, a_{3}\right) \sim\left(b_{1}, b_{2}, b_{3}{ }^{\prime}\right), \\
\left(a_{1}, a_{2}, a_{3}\right) \sim\left(b_{1}, b_{2}{ }^{\prime}, b_{3}\right), \\
\left(a_{1}, a_{2}, a_{3}{ }^{0}\right) \sim\left(a_{1}{ }^{\prime}, b_{2}, a_{3}{ }^{0}\right), \\
\left(a_{1}, a_{2}{ }^{0}, a_{3}\right) \sim\left(a_{1}{ }^{\prime}, a_{2}{ }^{0}, b_{3}\right) .
\end{gathered}
$$

Definition 9. A relation $\sim$ on $A_{1} \times A_{2} \times A_{3}$ satisfies the nonadditive weighting condition provided that for all $a_{1}, b_{1} \in A_{1}, c_{1} \in A_{1}-A_{1}{ }^{0}, d_{1} \in A_{1}-A_{1}^{00}, a_{2}, b_{2}, d_{2}$, $e_{2}, f_{2}, g_{2} \in A_{2}, c_{2} \in A_{2}-A_{2}{ }^{0}, a_{3}, b_{3}, d_{3}, e_{3}, f_{3}, g_{3} \in A_{3}$, and $c_{3} \in A_{3}-A_{3}{ }^{0}, c_{2} \approx g_{2}$, and $c_{3} \approx g_{3}$, if

then

$$
\begin{gathered}
\left(a_{1}, e_{2}, c_{3}\right) \sim\left(b_{1}, c_{2}, e_{3}\right), \\
\left(d_{1}, f_{2}, a_{3}\right) \sim\left(c_{1}, g_{2}, d_{3}\right), \\
\left(c_{1}, d_{2}, d_{3}\right) \sim\left(c_{1}, b_{2}, f_{3}\right), \\
\left(a_{1}, a_{2}, g_{3}\right) \sim\left(b_{1}, d_{2}, b_{3}\right), \\
\left(c_{1}, c_{2}, f_{3}\right) \sim\left(d_{1}, f_{2}, c_{3}\right), \\
\left(a_{1}, e_{2}, g_{3}\right) \sim\left(b_{1}, g_{2}, e_{3}\right), \\
\left(c_{1}, g_{2}, f_{3}\right) \sim\left(d_{1}, f_{2}, g_{3}\right),
\end{gathered}
$$

$$
\left(a_{1}, a_{2}, a_{3}\right) \sim\left(b_{1}, b_{2}, b_{3}\right) .
$$

This complicated property is necessary for all the simple models as well as for the nonsimple model. To show the necessity for the nonsimple model, $\omega_{1}\left(a_{1}\right) \phi_{2}\left(a_{2}\right)+$ $\omega_{2}\left(a_{1}\right) \phi_{3}\left(a_{3}\right)$, we translate the above equivalences into equations. We drop the notations for the functions and use $a_{1}$ to represent $\omega_{1}\left(a_{1}\right), a_{1}{ }^{\prime}$ to represent $\omega_{2}\left(a_{1}\right)$, and $a_{2}$ and $a_{3}$ to represent $\phi_{2}\left(a_{2}\right)$ and $\phi_{3}\left(a_{3}\right)$, respectively.

The fourth condition yields

$$
a_{1} a_{2}+a_{1}^{\prime} g_{3}=b_{1} d_{2}+b_{1}^{\prime} b_{3}
$$

The conclusion is that

$$
a_{1} a_{2}+a_{1}{ }^{\prime} a_{3}=b_{1} b_{2}+b_{1} b_{3} .
$$

Therefore, we need to show that the other six relations will provide

or

$$
a_{1}^{\prime} a_{3}+b_{1} d_{2}=b_{1} b_{2}+a_{1}^{\prime} g_{3}
$$

$$
a_{1}^{\prime}\left(a_{3}-g_{3}\right)=b_{1}\left(b_{2}-d_{2}\right) \text {. }
$$


The first and sixth condition can be translated into

and

$$
a_{1} e_{2}+a_{1}{ }^{\prime} c_{3}=b_{1} c_{2}+b_{1}{ }^{\prime} e_{3}
$$

$$
a_{1} e_{2}+a_{1}^{\prime} g_{3}=b_{1} g_{2}+b_{1}{ }^{\prime} e_{3} \text {. }
$$

Subtracting the latter from the former, we have

$$
a_{1}^{\prime}\left(c_{3}-g_{3}\right)=b_{1}\left(c_{2}-g_{2}\right) .
$$

Similarly, the second and seventh conditions will provide that

$$
c_{1}^{\prime}\left(d_{3}-f_{3}\right)=d_{1}^{\prime}\left(a_{3}-g_{3}\right) .
$$

The third relation can be translated and rewritten as

It follows that

$$
c_{1}\left(b_{2}-d_{2}\right)=c_{1}^{\prime}\left(d_{3}-f_{3}\right) .
$$

$$
d_{1}^{\prime}\left(a_{3}-g_{3}\right)=c_{1}\left(b_{2}-d_{2}\right) .
$$

The fifth and seven relations yield

$$
c_{1}\left(c_{2}-g_{2}\right)=d_{1}^{\prime}\left(c_{3}-g_{3}\right) .
$$

Multiplying (4.1), (4.2), and (4.3) together, we have

$$
a_{1}^{\prime} d_{1}^{\prime} c_{1}\left(c_{3}-g_{3}\right)\left(a_{3}-g_{3}\right)\left(c_{2}-g_{2}\right)=b_{1} c_{1} d_{1}^{\prime}\left(c_{2}-g_{2}\right)\left(b_{2}-d_{2}\right)\left(c_{3}-g_{3}\right) .
$$

Since $c_{1} \neq 0, d_{1}{ }^{\prime} \neq 0\left(c_{1}\right.$ is not in $A_{1}{ }^{0}$, and $d_{1}$ is not in $\left.A_{1}^{00}\right), c_{2} \neq g_{2}$, and $c_{3} \neq g_{3}$, wc cancel $c_{1} d_{1}^{\prime}\left(c_{2}-g_{2}\right)\left(c_{3}-g_{3}\right)$ and obtain

$$
a_{1}{ }^{\prime}\left(a_{3}-g_{3}\right)=b_{1}\left(b_{2}-d_{2}\right),
$$

as required.

From a practical point of view this property is too complicated to test directly. The conditions can be simplified by letting $e_{2}=f_{2}=g_{2}=a_{2}{ }^{0}$ and $e_{3}=f_{3}=g_{3}=a_{3}{ }^{0}$. The theorems which we want to obtain will not be affected by this simplification. The simplified condition is somewhat easier to test empirically.

We now want to list all the conditions which together are sufficient for the nonsimple model $\omega_{1}\left(a_{1}\right) \phi_{2}\left(a_{2}\right)+\omega_{2}\left(a_{1}\right) \phi_{3}\left(a_{3}\right)$.

It is clear that $\omega_{1}\left(a_{1}\right) \phi_{2}\left(a_{2}\right)+\omega_{2}\left(a_{1}\right) \phi_{3}\left(a_{3}\right)$ is an additive model for $\left\{a_{1}\right\} \times A_{2} \times A_{3}$. Therefore, we need conditions which are sufficient for the additive model. When $\phi_{3}\left(a_{3}\right)=0, \omega_{1}\left(a_{1}\right) \phi_{2}\left(a_{2}\right)$ is a multiplicative model for $A_{1} \times A_{2}$. Therefore, we need an axiom which states that $A_{1}$ and $A_{2} \times A_{3}{ }^{0}$ are mutually sign dependent. Similarly, $A_{1}$ and $A_{2}{ }^{0} \times A_{3}$ are mutually sign dependent, but this can be asserted by other 
axioms. Finally, the weighting condition functions in the nonsimple distributive model the same as the distributive cancellation axiom functions in the simple distributive model.

Definition 10. A relation $\geqslant$ on $A_{1} \times A_{2} \times A_{3}$ is a nonsimple distributive model iff it satisfies the following axioms.

Axiom 1. $\geqslant$ is a weak order.

Axiom 2. $A_{2}$ and $A_{3}$ are essential. $A_{2}{ }^{0}$ and $A_{3}{ }^{0}$ are not empty.

Axiom 3. $A_{1}$ and $A_{2} \times A_{3}{ }^{0}$ are mutually sign dependent.

Axiom 4. For any $a_{1} \in A_{1},\left\{a_{1}\right\} \times A_{2}$ and $\left\{a_{1}\right\} \times A_{3}$ are mutually independent.

Axiom 5. Unrestricted solvability of Definition 8 holds.

Axiom 6. For any induced order on $A_{2} \times A_{3}$, every strictly bounded standard sequence in one component is finite (Definition 7).

Axiom 7. The nonadditive weighting condition of Definition 9 holds.

\section{THEOREMS}

THEOREM 1. If a relation $\geqslant$ on $A_{1} \times A_{2} \times A_{3}$ is a nonsimple distributive model then the Thomsen condition holds.

Proof. The proof has to be done separately for the different conditions on the elements. The proof for a representative case is shown below.

Given $\left(a_{1}, a_{2}, k_{3}\right) \sim\left(a_{1}, k_{2}, b_{3}\right)$ and $\left(a_{1}, k_{2}, a_{3}\right) \sim\left(a_{1}, b_{2}, k_{\mathrm{g}}\right)$, we need to show that $\left(a_{1}, a_{2}, a_{3}\right) \sim\left(a_{1}, b_{2}, b_{3}\right)$.

We consider the case for which

$$
k_{2} \notin A_{2}{ }^{0}, k_{3} \notin A_{3}{ }^{0}, \quad \text { and } \quad a_{1} \notin A_{1}{ }^{0} \cup A_{1}^{00} .
$$

Since we have

and

$$
\begin{array}{ll}
\left(a_{1}, a_{2}, k_{3}\right) \sim\left(a_{1}, k_{2}, b_{3}\right) & \text { from the given condition, } \\
\left(a_{1}, k_{2}, a_{3}\right) \sim\left(a_{1}, k_{2}, a_{3}\right) & \text { from Axiom 1, } \\
\left(a_{1}, k_{2}, a_{3}\right) \sim\left(a_{1}, b_{2}, k_{3}\right) & \text { from the given condition, } \\
\left(a_{1}, a_{2}, k_{3}\right) \sim\left(a_{1}, k_{2}, b_{3}\right) & \text { from the given condition, } \\
\left(a_{1}, k_{2}, k_{3}\right) \sim\left(a_{1}, k_{2}, k_{3}\right) & \text { from Axiom 1, } \\
\left(a_{1}, a_{2}, k_{3}\right) \sim\left(a_{1}, k_{2}, b_{3}\right) & \text { from the given condition }
\end{array}
$$

$$
\left(a_{1}, k_{2}, k_{3}\right) \sim\left(a_{1}, k_{2}, k_{3}\right) \quad \text { from Axiom } 1,
$$


the nonadditive weighting condition implies that

$$
\left(a_{1}, a_{2}, a_{3}\right) \sim\left(a_{1}, b_{1}, b_{2}\right)
$$

THEOREM 2. If a relation $\geqslant$ on a set $A_{1} \times A_{2} \times A_{3}$ is a nonsimple distributive model, then there exist real-valued functions $\omega_{1}$ and $\omega_{2}$ on $A_{1}, \phi_{2}$ on $A_{2}$, and $\phi_{3}$ on $A_{3}$ such that

$$
\left(a_{1}, a_{2}, a_{3}\right) \geqslant\left(b_{1}, b_{2}, b_{3}\right)
$$

iff

$$
\omega_{1}\left(a_{1}\right) \phi_{2}\left(a_{2}\right)+\omega_{2}\left(a_{1}\right) \phi_{3}\left(a_{3}\right) \geqslant \omega_{1}\left(b_{1}\right) \phi_{2}\left(b_{2}\right)+\omega_{2}\left(b_{1}\right) \phi_{3}\left(a_{3}\right)
$$

for all $\left(a_{1}, a_{2}, a_{3}\right)$ and $\left(b_{1}, b_{2}, b_{3}\right) \in A_{1} \times A_{2} \times A_{3}$.

Moreover, the real value functions satisfying this property are unique up to the following transformations.

$$
\begin{aligned}
& \omega_{1}\left(a_{1}\right) \rightarrow \alpha_{1} \omega_{1}^{\prime}\left(a_{1}\right), \\
& \omega_{2}\left(a_{1}\right) \rightarrow \alpha_{2} \omega_{2}^{\prime}\left(a_{1}\right), \\
& \phi_{2}\left(a_{2}\right) \rightarrow \beta_{2} \phi_{2}^{\prime}\left(a_{2}\right), \\
& \phi_{3}\left(a_{3}\right) \rightarrow \beta_{3} \phi_{3}^{\prime}\left(a_{3}\right),
\end{aligned}
$$

where $\alpha_{1} \beta_{2}=\alpha_{2} \beta_{3}>0$.

Proof. For the case in which $A_{1} \neq A_{1}{ }^{0} \cup A_{1}^{00}$, we define two operations $\oplus$ and $*$. We will then prove that there is an isomorphism from $\left\langle A_{1} \times A_{2} \times A, \geqslant, \oplus, *\right\rangle$ to a subring of $\langle R, \geqslant,+, \cdot\rangle$, provided that Axioms 1-7 hold. The representation theorem for the case in which $A_{1}=A_{1}{ }^{0} \cup A_{1}^{00}$ will be discussed at the end.

Let $\left(\mathbf{a}_{1}, \mathbf{a}_{2}, \mathbf{a}_{3}\right)$ denote the equivalence class of $\left(a_{1}, a_{2}, a_{3}\right)$ for all $\left(a_{1}, a_{2}, a_{3}\right) \in$ $A_{1} \times A_{2} \times A_{3}$. Clearly, if $\left(a_{1}, a_{2}, a_{3}\right) \sim\left(b_{1}, b_{2}, b_{3}\right)$, then the equivalence class of $\left(a_{1}, a_{2}, a_{3}\right)$ is exactly the same as the equivalence class of $\left(b_{1}, b_{2}, b_{3}\right)$, i.e., $\left(\mathbf{a}_{1}, \mathbf{a}_{2}, \mathbf{a}_{3}\right)=\left(\mathbf{b}_{1}, \mathbf{b}_{2}, \mathbf{b}_{3}\right)$. Let $\geqslant$ denote the order relation on $A_{1} \times A_{2} \times A_{3} / \sim$, and $\left(\mathrm{a}_{1}, \mathrm{a}_{2}, \mathrm{a}_{3}\right) \geqslant\left(\mathrm{b}_{1}, \mathrm{~b}_{2}, \mathrm{~b}_{3}\right)$ if and only if $\left(a_{1}, a_{2}, a_{3}\right) \geqslant\left(b_{1}, b_{2}, b_{3}\right)$. Define $\mathbf{a}=\left(\mathbf{a}_{1}, \mathbf{a}_{2}, \mathbf{a}_{3}\right)$, and $\boldsymbol{a}=\left(a_{1}, a_{2}, a_{3}\right)$. We let $\mathbf{a}>\mathbf{b}$ represent $\mathbf{a} \geqslant \mathbf{b}$ and not $\mathbf{b} \geqslant \mathbf{a}$. Clearly, the relation $\geqslant$ on the equivalence classes is a simple order.

Select $a_{1}{ }^{i}, a_{1}^{i i} \in A_{1}-A_{1}^{0}-A_{1}^{00}, a_{2}^{i} \in A_{2}-A_{2}{ }^{0}, a_{3}{ }^{i} \in A_{3}-A_{3}{ }^{0}$, such that $\left(a_{1}^{i}, a_{2}{ }^{i}, a_{3}{ }^{0}\right) \sim\left(a_{1}^{i i}, a_{2}{ }^{0}, a_{3}{ }^{i}\right)$. For any $a \in A_{1} \times A_{2} \times A_{3}$, there exist $a_{1}^{\prime}, a_{1}^{\prime \prime} \in A_{1}$ such that $a \sim\left(a_{1}{ }^{\prime}, a_{2}{ }^{i}, a_{3}{ }^{0}\right) \sim\left(a_{1}^{\prime \prime}, a_{2}{ }^{0}, a_{3}{ }^{i}\right)$; and there exist $a_{2}{ }^{\prime} \in A_{2}$ and $a_{3}{ }^{\prime} \in A_{3}$ such that $a \sim\left(a_{1}{ }^{i}, a_{2}{ }^{\prime}, a_{3}{ }^{0}\right) \sim\left(a_{1}^{i i}, a_{2}{ }^{0}, a_{3}{ }^{\prime}\right)$ (using unrestricted solvability axiom). There also exist $a_{2}^{\prime \prime}$ of $A_{2}$ and $a_{3}^{\prime \prime}$ of $A_{3}$ such that $a \sim\left(a_{1}, a_{2}^{\prime \prime}, a_{3}{ }^{0}\right) \sim\left(a_{1}, a_{2}{ }^{0}, a_{3}^{\prime \prime}\right)$. 
We now define the operations $\oplus, *_{1}$, and $*_{2}$ as follows.

$$
\begin{aligned}
& \left(a_{1}, a_{2}, a_{3}^{0}\right) \oplus\left(a_{1}, a_{2}^{0}, a_{3}\right)=\left(a_{1}, a_{2}, a_{3}\right), \\
& \left(a_{1}, a_{2}{ }^{1}, a_{3}^{0}\right) *_{1}\left(a_{1}{ }^{i}, a_{2}, a_{3}{ }^{0}\right)=\left(a_{1}, a_{2}, a_{3}^{0}\right), \\
& \left(a_{1}, \mathbf{a}_{2}^{0}, a_{3}{ }^{i}\right) *_{2}\left(a_{1}^{11}, a_{2}^{0}, a_{3}\right)=\left(a_{1}, a_{2}^{0}, a_{3}\right) .
\end{aligned}
$$

It is necessary to show that $*_{1}=*_{2}=*$ first.

Step 1. We are going to show that $\mathbf{a} *_{1} \mathbf{b}=\mathbf{a} *_{2} \mathbf{b}$ for all $\mathbf{a}, \mathbf{b} \in A_{1} \times A_{2} \times A_{3} / \sim$.

The unrestricted solvability axiom quarantees that there exist $a_{1} \in A_{1}$ and $a_{3} \in A_{3}$ such that $\left(a_{1}, a_{2}{ }^{0}, a_{3}{ }^{i}\right)$ is in the equivalence class $a$ and $\left(a_{1}^{i i}, a_{2}{ }^{0}, b_{3}\right)$ is in the equivalence $\mathbf{b}$, or $\mathbf{a}=\left(\mathbf{a}_{1}, \mathbf{a}_{2}{ }^{0}, \mathbf{a}_{\mathbf{3}}{ }^{1}\right)$ and $\mathbf{b}=\left(\mathbf{a}_{\mathbf{1}}^{\mathbf{i}}, \mathbf{a}_{\mathbf{2}}{ }^{\mathbf{0}}, \mathbf{b}^{\mathbf{3}}\right)$. Similarly, we can find $a_{1}{ }^{\prime} \in A_{1}$, $b_{2} \in A_{2}$, and $b_{3}{ }^{\prime} \in A_{3}$ such that

$$
\begin{gathered}
\left(a_{1}, a_{2}{ }^{0}, a_{3}{ }^{i}\right) \sim\left(a_{1}{ }^{\prime}, a_{2}{ }^{i}, a_{3}{ }^{0}\right), \\
\left(a_{1}^{i i}, a_{2}{ }^{0}, b_{3}\right) \sim\left(a_{1}{ }^{i}, a_{2}{ }^{0}, b_{3}{ }^{\prime}\right), \\
\left(a_{1}{ }^{i}, a_{2}{ }^{0}, b_{3}{ }^{\prime}\right) \sim\left(a_{1}{ }^{i}, b_{2}, a_{3}{ }^{0}\right) .
\end{gathered}
$$

But

$$
\begin{aligned}
& \left(a_{1}, a_{2}{ }^{0}, a_{3}{ }^{0}\right) \sim\left(a_{i}{ }^{\prime}, a_{2}{ }^{0}, a_{3}{ }^{0}\right), \\
& \left(a_{1}^{i}, a_{2}{ }^{i}, a_{3}{ }^{0}\right) \sim\left(a_{1}^{i i}, a_{2}{ }^{0}, a_{3}{ }^{i}\right), \\
& \left(a_{1}, a_{2}{ }^{0}, a_{3}{ }^{0}\right) \sim\left(a_{1}{ }^{\prime}, a_{2}{ }^{0}, a_{3}{ }^{0}\right), \\
& \left(a_{1}{ }^{i}, a_{2}{ }^{0}, a_{3}{ }^{0}\right) \sim\left(a_{1}^{i i}, a_{2}{ }^{0}, a_{3}{ }^{0}\right) .
\end{aligned}
$$

Applying Axiom 7 to the above equations, we have

$$
\left(a_{1}, a_{2}^{0}, b_{3}\right) \sim\left(a_{1}{ }^{\prime}, b_{2}, a_{3}^{0}\right) .
$$

Because $a=\left(a_{1}, a_{2}{ }^{0}, a_{3}{ }^{i}\right)=\left(a_{1}^{\prime}, a_{2}{ }^{1}, a_{3}{ }^{0}\right)$ and $b=\left(a_{1}^{i i}, a_{2}{ }^{0}, b_{3}\right)=\left(a_{1}{ }^{1}, b_{2}, a_{3}{ }^{0}\right)$, $\mathbf{a} *_{2} \mathbf{b}=\left(\mathbf{a}_{1}, \mathbf{a}_{2}{ }^{0}, \mathbf{b}_{3}\right)$ and $\mathbf{a} *_{1} \mathbf{b}=\left(\mathbf{a}_{1}{ }^{\prime}, \mathbf{b}_{2}, \mathbf{a}_{3}{ }^{0}\right)$. We just prove that $\left(\mathbf{a}_{1}{ }^{\prime}, \mathbf{b}_{2}, \mathbf{a}_{3}{ }^{0}\right)=$ $\left(a_{1}, a_{2}{ }^{0}, b_{3}\right)$, that is $\mathbf{a} *_{1} \mathbf{b}=\mathbf{a} *_{2} \mathbf{b}$.

We are now going to show that $\left\langle A_{1} \times A_{2} \times A_{3} / \sim, \geqslant, \oplus, *\right\rangle$ is an Archimedean ordered ring. In other words, we need to show four things:

(a) $\left\langle A_{1} \times A_{2} \times A_{3} / \sim, \geqslant, \oplus\right\rangle$ is an Archimedean ordered group,

(b) $*$ is associative,

(c) $*$ is distributive to $\oplus$,

(d) there is a zero element $\theta$, if $\mathbf{a}>\theta$ and $\mathbf{b}>\mathbf{c}$, then $\mathbf{a} * \mathbf{b}>\mathbf{a} * \mathbf{c}$ and $\mathbf{b} * \mathbf{a} \succ \mathbf{c} * \mathbf{a}$.

Step 2. The proof of part $a$ is contained in Krantz et al. (1971, pp. 257-266). 
Step 3. For any $\mathbf{a}, \mathbf{b}, \mathbf{c} \in A_{1} \times A_{2} \times A_{3} / \sim$, we will prove that $\mathbf{a} *(\mathbf{b} * \mathbf{c})=$ $(\mathbf{a} * \mathbf{b}) * \mathbf{c}$. Let

$$
\begin{aligned}
& \mathbf{a}=\left(\mathbf{a}_{1}, \mathbf{a}_{2}{ }^{\mathrm{i}}, \mathbf{a}_{3}{ }^{0}\right), \\
& \mathbf{b}=\left(\mathbf{b}_{1}, \mathbf{a}_{2}{ }^{0}, \mathbf{a}_{3}{ }^{\mathrm{i}}\right), \\
& \mathbf{c}=\left(\mathbf{a}_{1}^{\mathrm{i}}, \mathbf{a}_{2}{ }^{0}, \mathbf{c}_{3}\right),
\end{aligned}
$$

The unrestricted solvability axiom provides a $b_{2} \in A_{2}$ such that $\left(b_{1}, a_{2}{ }^{0}, a_{3}{ }^{i}\right) \sim$ $\left(a_{1}{ }^{i}, b_{2}, a_{3}{ }^{0}\right)$. By unrestricted solvability again, we have the following relations.

but

$$
\begin{aligned}
& \left(a_{1}{ }^{\prime}, a_{2}{ }^{0}, a_{3}{ }^{i}\right) \sim\left(a_{1}, b_{2}, a_{3}{ }^{0}\right), \quad \text { for an } a_{1}{ }^{\prime} \in A_{1} \text {, } \\
& \left(b_{1}, a_{2}{ }^{0}, c_{3}\right) \sim\left(a_{1}{ }^{i}, a_{2}{ }^{0}, c_{3}{ }^{\prime}\right), \quad \text { for a } \quad c_{3}{ }^{\prime} \in A_{3} \text {, } \\
& \left(a_{1}{ }^{i}, a_{2}{ }^{0}, c_{3}{ }^{\prime}\right) \sim\left(a_{1}{ }^{i}, c_{2}, a_{3}{ }^{0}\right), \quad \text { for a } \quad c_{2} \in A_{2} \text {, }
\end{aligned}
$$

$$
\begin{aligned}
& \left(a_{1}{ }^{\prime}, a_{2}{ }^{0}, a_{3}{ }^{0}\right) \sim\left(a_{1}, a_{2}{ }^{0}, a_{3}{ }^{0}\right), \\
& \left(a_{1}{ }^{i}, b_{2}, a_{3}{ }^{0}\right) \sim\left(b_{1}, a_{2}{ }^{0}, a_{3}{ }^{i}\right) .
\end{aligned}
$$

From definition 3, we have

$$
\begin{aligned}
& \left(a_{1}{ }^{1}, a_{2}{ }^{0}, a_{3}{ }^{0}\right) \sim\left(a_{1}, a_{2}{ }^{0}, a_{3}{ }^{0}\right), \\
& \left(a_{1}{ }^{i}, a_{2}{ }^{0}, a_{3}{ }^{0}\right) \sim\left(b_{1}, a_{2}{ }^{0}, a_{3}{ }^{0}\right) .
\end{aligned}
$$

Applying Axiom 7 to the above seven formulas, it results

$$
\left(a_{1}{ }^{\prime}, a_{2}{ }^{0}, c_{3}\right) \sim\left(a_{1}, c_{2}, a_{3}{ }^{0}\right) .
$$

From the definitions of $*$, we have $\mathbf{b} * \mathbf{c}=\left(\mathbf{b}_{1}, \mathbf{a}_{2}{ }^{0}, \mathbf{c}_{3}\right)$. From the transitive property of the equivalence relation $\sim, b * c=\left(a_{1}{ }^{1}, c_{2}, a_{3}{ }^{0}\right)$. Therefore, $\mathbf{a} *(\mathbf{b} * \mathbf{c})=$ $\left(a_{1}, c_{2}, a_{3}{ }^{0}\right)$. Similarly, $a * b=\left(a_{1}, b_{2}, a_{3}{ }^{0}\right)=\left(a_{1}{ }^{\prime}, a_{2}{ }^{0}, a_{3}{ }^{1}\right)$, and hence $(a * b) * c=$ $\left(\mathbf{a}_{1}{ }^{\prime}, \mathbf{a}_{2}{ }^{0}, \mathbf{c}_{3}\right)$. We proved previously that $\left(a_{1}{ }^{\prime}, a_{2}{ }^{0}, c_{3}\right) \sim\left(a_{1}, c_{2}, a_{3}{ }^{0}\right)$, consequently, we have $(\mathrm{a} * \mathrm{~b}) * \mathrm{c}=\mathbf{a} *(\mathrm{~b} * \mathrm{c})$.

Step 4. We will now prove that $*$ is distributive to $\oplus$. In other words, it has to be shown that $\mathbf{a} *(\mathbf{b} \oplus \mathbf{c})=(\mathbf{a} * \mathbf{b}) \oplus(\mathbf{a} * \mathbf{c})$ for all $\mathbf{a}, \mathbf{b}, \mathbf{c} \in A_{1} \times A_{2} \times A_{3} / \sim$. Because of Axiom 5, we can always let $a=\left(a_{1}, a_{2}{ }^{0}, a_{3}{ }^{1}\right), b=\left(a_{1}{ }^{1}, b_{2}, a_{3}{ }^{0}\right)$ and $\mathbf{c}=\left(\mathbf{a}_{1}^{\mathbf{i}}, \mathbf{a}_{\mathbf{2}}{ }^{0}, \mathbf{c}_{3}\right)$. By the same axiom, we have the following relations:

$$
\begin{array}{rll}
\left(a_{1}, a_{2}{ }^{0}, a_{3}{ }^{i}\right) \sim\left(a_{1}{ }^{\prime}, a_{2}{ }^{i}, a_{3}{ }^{0}\right), & \text { for an } & a_{1}{ }^{\prime} \in A_{1}, \\
\left(a_{1}^{i}, a_{2}{ }^{0}, c_{3}\right) \sim\left(a_{1}{ }^{i}, a_{2}{ }^{0}, x_{3}\right), & \text { for a } & x_{3} \in A_{3}, \\
\left(a_{1}{ }^{i}, b_{2}, x_{3}\right) \sim\left(a_{1}{ }^{\prime}, b_{2}{ }^{\prime}, a_{3}{ }^{0}\right), & \text { for a } & b_{2}{ }^{\prime} \in A_{2}, \\
\left(a_{1}{ }^{\prime}, b_{2}, a_{3}{ }^{0}\right) \sim\left(a_{1}, b_{2}^{\prime \prime}, a_{3}{ }^{0}\right), & \text { for a } & b_{2}^{\prime \prime} \in A_{2} .
\end{array}
$$


It was chosen that

$$
\begin{aligned}
& \left(a_{1}^{i}, a_{2}^{i}, a_{3}{ }^{0}\right) \sim\left(a_{1}^{i i}, a_{2}{ }^{0}, a_{3}{ }^{i}\right), \\
& \left(a_{1}, a_{2}{ }^{0}, a_{3}{ }^{0}\right) \sim\left(a_{1}{ }^{\prime}, a_{2}{ }^{0}, a_{3}{ }^{0}\right), \\
& \left(a_{1}{ }^{i}, a_{2}{ }^{0}, a_{3}{ }^{0}\right) \sim\left(a_{1}^{i i}, a_{2}{ }^{0}, a_{3}{ }^{0}\right) .
\end{aligned}
$$

Again, Axiom 7 implies

$$
\left(a_{1}, b_{2}^{\prime \prime}, c_{3}\right) \sim\left(a_{1}^{\prime}, b_{2}^{\prime}, a_{3}{ }^{0}\right)
$$

Since $c=\left(\mathbf{a}_{1}^{\mathbf{i}}, \mathbf{a}_{2}{ }^{0}, \mathbf{c}_{3}\right)=\left(\mathbf{a}_{1}{ }^{1}, \mathbf{a}_{2}{ }^{0}, \mathbf{x}_{3}\right)$, it follows from the definition $\oplus$ that $\mathbf{b} \oplus \mathbf{c}=\left(\mathrm{a}_{1}{ }^{1}, \mathrm{~b}_{2}, \mathrm{x}_{3}\right)$. But $\left(\mathrm{a}_{1}{ }^{1}, \mathrm{~b}_{2}, \mathbf{x}_{3}\right)=\left(\mathrm{a}_{1}{ }^{1}, \mathrm{~b}_{2}{ }^{\prime}, \mathrm{a}_{3}{ }^{0}\right)$, and $\mathbf{a}=\left(\mathrm{a}_{1}, \mathrm{a}_{2}{ }^{0}, \mathrm{a}_{3}{ }^{1}\right)=$ $\left(a_{1}{ }^{\prime}, a_{2}{ }^{1}, a_{3}{ }^{0}\right)$, hence $a *(b \oplus c)=\left(a_{1}{ }^{\prime}, b_{2}{ }^{\prime}, a_{3}{ }^{0}\right)$. Clearly, $\mathbf{a} * \mathbf{c}=\left(\mathbf{a}_{1}, \mathbf{a}_{2}{ }^{0}, \mathbf{c}_{3}\right)$, and $\mathbf{a} * \mathbf{b}=\left(\mathbf{a}_{1}{ }^{\prime}, \mathbf{b}_{\mathbf{2}}, \mathbf{a}_{3}{ }^{0}\right)=\left(\mathbf{a}_{1}, \mathbf{b}_{\mathbf{2}}^{\prime \prime}, \mathbf{a}_{\mathbf{3}}{ }^{0}\right)$, the definition of $\oplus$ provides that $(\mathbf{a} * \mathbf{b}) \oplus(\mathbf{a} * \mathbf{c})=\left(\mathbf{a}_{1}, \mathbf{b}_{2}^{\prime \prime}, \mathbf{c}_{3}\right)$. Since $\left(a_{1}, b^{\prime \prime}, c_{3}\right) \sim\left(a_{1}{ }^{\prime}, b_{2}{ }^{\prime}, a_{3}{ }^{0}\right)$, it follows that $(\mathbf{a} * \mathbf{b}) \oplus(\mathbf{a} * \mathbf{c})=\mathbf{a} *(\mathbf{b} \oplus \mathbf{c})$.

We thus proved that $\left\langle A_{1} \times A_{2} \times A_{3} / \sim, \oplus, *\right\rangle$ is a ring with zero element $\theta=\left(\mathbf{a}_{1}, \mathbf{a}_{\mathbf{2}}{ }^{\mathbf{0}}, \mathbf{a}_{\mathbf{3}}{ }^{0}\right)$. It is easy to show that Axiom 3 leads to the conclusion that $\mathbf{a}>\theta$ and $\mathbf{b}>\mathbf{c}$ imply $\mathbf{a} * \mathbf{b}>\mathbf{a} * \mathbf{c}$ and $\mathbf{b} * \mathbf{a}>\mathbf{c} * \mathbf{a}$. We thereby conclude that $\left\langle A_{1} \times A_{2} \times A_{3} / \sim, \geqslant, \oplus, *\right\rangle$ is an Archimedean ordered ring.

An Archimedean ordered ring is uniquely isomorphic to a subring of $\langle R, \geqslant,+, \cdot\rangle$ (Krantz et al., 1971, p. 58). Hence there exists a function $\Psi$ such that

$$
\begin{gathered}
\mathbf{a} \geqslant \mathbf{b} \quad \text { iff } \quad \Psi(\mathbf{a}) \geqslant \Psi(\mathbf{b}), \\
\Psi(\mathbf{a} \oplus \mathbf{b})=\Psi(\mathbf{a})+\Psi(\mathbf{b}), \\
\Psi(\mathbf{a} * \mathbf{b})=\Psi(\mathbf{a}) \cdot \Psi(\mathbf{b}) .
\end{gathered}
$$

By the definitions of $\oplus$ and $*$, we have

$$
\begin{aligned}
\left(\mathbf{a}_{1}, \mathbf{a}_{2}, \mathbf{a}_{3}\right)= & \left(\mathbf{a}_{1}, \mathbf{a}_{2}{ }^{1}, \mathbf{a}_{3}{ }^{0}\right) *\left(\mathbf{a}_{1}{ }^{1}, \mathbf{a}_{2}, \mathbf{a}_{3}{ }^{0}\right) \\
& \left(\oplus\left(\mathbf{a}_{1}, \mathbf{a}_{2}{ }^{0}, \mathbf{a}_{3}{ }^{1}\right) *\left(\mathbf{a}_{1}^{11}, \mathbf{a}_{2}{ }^{0}, \mathbf{a}_{3}\right) .\right.
\end{aligned}
$$

Therefore, we have the following relation:

$$
\begin{aligned}
\Psi\left(\mathbf{a}_{1}, \mathbf{a}_{2}, \mathbf{a}_{3}\right)= & \Psi\left(\mathbf{a}_{1}, \mathbf{a}_{2}{ }^{1}, \mathbf{a}_{3}{ }^{0}\right) \Psi\left(\mathbf{a}_{1}{ }^{1}, \mathbf{a}_{2}, \mathbf{a}_{3}{ }^{0}\right) \\
& \mid \Psi\left(\mathbf{a}_{1}, \mathbf{a}_{2}{ }^{0}, \mathbf{a}_{3}{ }^{1}\right) \Psi\left(\mathbf{a}_{1}^{11}, \mathbf{a}_{2}{ }^{0}, \mathbf{a}_{3}\right) .
\end{aligned}
$$

Let us define $\omega_{1}$ and $\omega_{2}, \phi_{2}$ and $\phi_{3}$ such that

$$
\begin{array}{lll}
\omega_{1}\left(a_{1}\right)=\Psi\left(\mathbf{a}_{1}, \mathbf{a}_{2}{ }^{1}, \mathbf{a}_{3}{ }^{0}\right), & \text { for all } & a_{1} \in A_{1}, \\
\omega_{2}\left(a_{1}\right)=\Psi\left(\mathbf{a}_{1}, \mathbf{a}_{2}{ }^{0}, \mathbf{a}_{3}{ }^{1}\right), & \text { for all } & a_{1} \in A_{1}, \\
\phi_{2}\left(a_{2}\right)=\Psi\left(\mathbf{a}_{1}{ }^{1}, \mathbf{a}_{2}, \mathbf{a}_{3}{ }^{0}\right), & \text { for all } & a_{2} \in A_{2}, \\
\phi_{3}\left(a_{3}\right)=\Psi\left(\mathbf{a}_{1}, \mathbf{a}_{2}{ }^{0}, \mathbf{a}_{3}\right), & \text { for all } & a_{3} \in A_{3} .
\end{array}
$$


Finally, we have

$$
a \geqslant b \quad \text { iff } \quad \omega_{1}\left(a_{1}\right) \phi_{2}\left(a_{2}\right)+\omega_{2}\left(a_{1}\right) \phi_{3}\left(a_{3}\right) \geqslant \omega_{1}\left(b_{1}\right) \phi_{2}\left(b_{2}\right)+\omega_{2}\left(b_{1}\right) \phi_{3}\left(b_{3}\right) .
$$

Step 5. If $\omega_{1}{ }^{\prime}, \omega_{2}{ }^{\prime}, \phi_{2}{ }^{\prime}$, and $\phi_{3}{ }^{\prime}$ are any other functions satisfying the above equation, we have the following relation

$$
\omega_{1}\left(a_{1}\right) \phi_{2}\left(a_{2}\right)+\omega_{2}\left(a_{1}\right) \phi_{3}\left(a_{3}\right)=\alpha\left[\omega_{1}^{\prime}\left(a_{1}\right) \phi_{2}^{\prime}\left(a_{2}\right)+\omega_{2}^{\prime}\left(a_{1}\right) \phi_{3}{ }^{\prime}\left(a_{3}\right)\right]
$$

for all $\left(a_{1}, a_{2}, a_{3}\right) \in\left\{a_{1}\right\} \times A_{2} \times A_{3}$, where $\alpha$ is a positive constant (see Krantz, Luce, Suppes, and Tversky, 1971, p. 257).

Let $a_{3}=a_{3}{ }^{0}$, we have $\phi_{2}\left(a_{2}\right)=\alpha \omega_{1}{ }^{\prime}\left(a_{1}\right) \phi_{2}{ }^{\prime}\left(a_{2}\right) / \omega_{1}\left(a_{1}\right)$ and

$$
\phi_{2}\left(a_{2}^{i}\right)=\alpha \omega_{1}^{\prime}\left(a_{1}\right) \phi_{2}^{\prime}\left(a_{2}^{i}\right) / \omega_{1}\left(a_{1}\right) .
$$

It follows that $\phi_{2}\left(a_{2}\right)=\left[\phi_{2}\left(a_{2}{ }^{i}\right) / \phi_{2}{ }^{\prime}\left(a_{2}{ }^{i}\right)\right] \phi_{2}{ }^{\prime}\left(a_{2}\right)$. Similarly, it can be shown that $\phi_{3}\left(a_{3}\right)=\left[\phi_{3}\left(a_{3}{ }^{i}\right) / \phi_{3}{ }^{\prime}\left(a_{3}{ }^{i}\right)\right] \phi_{3}{ }^{\prime}\left(a_{3}\right)$.

We now want to show that $\omega_{1}\left(a_{1}\right)=\left[\omega_{1}\left(a_{1}^{i}\right) / \omega_{1}{ }^{\prime}\left(a_{1}^{i}\right)\right] \omega_{1}{ }^{\prime}\left(a_{1}\right)$. Unrestrictive solvability guarantees that there exist $b_{2} \in A_{2}$ such that

$$
\left(a_{1}, a_{2}, a_{3}{ }^{0}\right) \sim\left(a_{1}^{i}, b_{2}, a_{3}^{0}\right) .
$$

It implies that $\omega_{1}\left(a_{1}\right) \phi_{2}\left(a_{2}\right)=\omega_{1}\left(a_{1}{ }^{i}\right) \phi_{2}\left(b_{2}\right)$ and $\omega_{1}^{\prime}\left(a_{1}\right) \phi_{2}{ }^{\prime}\left(a_{2}\right)=\omega_{1}^{\prime}\left(a_{1}{ }^{i}\right) \phi_{2}{ }^{\prime}\left(b_{2}\right)$. But $\omega_{1}\left(a_{1}\right) \phi_{2}\left(a_{2}\right)=\alpha \omega_{1}^{\prime}\left(a_{1}\right) \phi_{2}{ }^{\prime}\left(a_{2}\right)$, hence $\omega_{1}\left(a_{1}^{i}\right) \phi_{2}\left(b_{2}\right)=\alpha \omega_{1}^{\prime}\left(a_{1}{ }^{i}\right) \phi_{2}{ }^{\prime}\left(b_{2}\right)$. Since $\phi_{2}\left(x_{2}\right)=\left[\phi_{2}\left(a_{2}{ }^{i}\right) / \phi_{2}{ }^{\prime}\left(a_{2}{ }^{i}\right)\right] \phi_{2}{ }^{\prime}\left(x_{2}\right)$ for either $x_{2}=a_{2}$ or $x_{2}=b_{2}$, it follows that $\omega_{1}\left(a_{1}\right)=\alpha\left[\phi_{2}{ }^{\prime}\left(a_{2}{ }^{i}\right) / \phi_{2}\left(a_{2}{ }^{i}\right)\right] \omega_{1}{ }^{\prime}\left(a_{1}\right)$ and $\omega_{1}\left(a_{1}{ }^{i}\right)=\alpha\left[\phi_{2}{ }^{\prime}\left(a_{2}{ }^{i}\right) / \phi_{2}\left(a_{2}{ }^{i}\right)\right] \omega_{1}{ }^{\prime}\left(a_{1}{ }^{i}\right)$. Consequently, we have $\omega_{1}\left(a_{1}\right)=\left[\omega_{1}\left(a_{1}^{i}\right) / \omega_{1}^{\prime}\left(a_{1}^{i}\right)\right] \omega_{1}^{\prime}\left(a_{1}\right)$.

By the similar procedure, it can be shown that $\omega_{2}\left(a_{1}\right)=\left[\omega_{2}\left(a^{i i}\right) / \omega_{2}{ }^{\prime}\left(a^{i i}\right)\right] \omega_{2}{ }^{\prime}\left(a_{1}\right)$. Let $\alpha_{1}=\omega_{1}\left(a_{1}^{i}\right) / \omega_{1}{ }^{\prime}\left(a_{1}{ }^{i}\right), \alpha_{2}=\omega_{2}\left(a^{i i}\right) / \omega_{2}{ }^{\prime}\left(a^{i i}\right), \beta_{2}=\phi_{2}\left(a_{2}{ }^{i}\right) / \phi_{2}{ }^{\prime}\left(a_{2}{ }^{i}\right)$, and $\alpha_{3}=\phi_{3}\left(a_{3}{ }^{i}\right) / \phi_{3}{ }^{\prime}\left(a_{3}{ }^{i}\right)$. Clearly, we have $\alpha_{1} \beta_{2}=\alpha_{2} \beta_{3}=\alpha>0$.

Therefore,

$$
\begin{aligned}
\omega_{1}^{\prime}\left(a_{1}\right) & =\alpha_{1} \omega_{1}{ }^{\prime}\left(a_{1}\right), \\
\omega_{2}\left(a_{1}\right) & =\alpha_{2} \omega_{2}^{\prime}\left(a_{1}\right), \\
\phi_{2}\left(a_{2}\right) & =\beta_{2} \phi_{2}{ }^{\prime}\left(a_{2}\right), \\
\phi_{3}\left(a_{3}\right) & =\beta_{3} \phi_{3}{ }^{\prime}\left(a_{3}\right),
\end{aligned}
$$

where $\alpha_{1} \beta_{2}=\alpha_{2} \beta_{3}>0$.

We now consider a special case in which $A_{1}=A_{1}{ }^{0} \cup A_{1}^{00}$. Since $A_{2}$ is essential, there are $a_{2}, b_{2} \in A_{2}$ such that $\left(a_{1}, a_{2}, a_{3}\right) \nsim\left(a_{1}, b_{2}, a_{3}\right)$ for some $a_{1} \in A_{1}$ and $a_{3} \in A_{3}$. If $a_{1} \in A_{1}{ }^{0}$, from definition of $A_{1}{ }^{0}$ we know that $\left(a_{1}, a_{2}, a_{3}\right) \sim\left(a_{1}, b_{2}, a_{3}\right)$. Therefore, $a_{1}$ must be in $A_{1}^{00}$. Clearly, there is no $b_{3} \in A_{3}$ such that $\left(a_{1}, a_{2}, a_{3}\right) \sim$ $\left(a_{1}, b_{2}, b_{3}\right)$. Hence the unrestricted solvability is not satisfied, and $\geqslant$ on $A_{1} \times A_{2} \times A_{3}$ is not a nonsimple distributed model. 


\section{ConClusions}

The weighting condition of Definition 9 provides a crucial test of the nonsimple distributive model against other nonsimple models. However, it cannot discriminate nonsimple models from the simple models. The weighting condition is also a necessary condition for the simple models presented by Krantz et al. (1971). It is the sign dependence which can diagnose the nonsimple models from the simple models. The nonsimple models satisfy neither joint independence nor proper sign dependence for each pair of factors. The solvability axiom of the nonsimple model is weaker than the solvability axiom of the simple models. For any $a_{1} \in A_{1}, a_{2}, b_{2} \in A_{2}, a_{3}, b_{3} \in A_{3}$, the unrestricted solvability axiom of the simple models guarantees that there exist $b_{1} \in A_{1}$ such that $\left(a_{1}, a_{2}, a_{3}\right) \sim\left(b_{1}, b_{2}, b_{3}\right)$. However the solvability axiom of the nonsimple model does not imply the existence of $b_{1}$.

The major difference between the nonsimple distributive model and the simple distributive model is on the different multiplicative effects of $A_{1}$ with $A_{2}$ and $A_{3}$. In the simple distributive model, the multiplicative effect of $A_{1}$ on $A_{2}$ is the same as the multiplicative effect of $A_{1}$ on $A_{3}$. Therefore, there is only one identity element of $A_{1}$. In the nonsimple distributive model, the multiplicative effect of $A_{1}$ on $A_{2}$ may be different from the multiplicative effect of $A_{1}$ on $A_{3}$. Therefore we need two identity elements $a_{1}{ }^{i}$ and $a_{1}^{i i}$ of $A_{1}$ which in turn restrict the uniqueness character of the real-valued functions of $A_{2}$ and $A_{3}$. In the distributive model, the real-valued functions of $A_{2}$ and $A_{3}$ are unique up to the interval scale, whereas in the nonsimple model the functions are unique up to the ratio scale.

The key concept in this paper is the multiple identity elements for one factor. This concept will allow the construction of axiom systems for the more complicated models.

\section{REFERENCE}

Krantz, D. H., Luce, R. D., Suppes, P., \& Tversky, A. Foundations of measurement. Vol. I. New York: Academic Press, 1971.

ReCEIVED: May 21, 1974 